\title{
Fermi-Pasta-Ulam Recurrence in Nonlinear Fiber Optics: The Role of Reversible and Irreversible Losses
}

\author{
Arnaud Mussot, ${ }^{1, *}$ Alexandre Kudlinski, ${ }^{1}$ Maxime Droques, ${ }^{1}$ Pascal Szriftgiser, ${ }^{1}$ and Nail Akhmediev ${ }^{2}$ \\ ${ }^{1}$ Laboratoire PhLAM UMR CNRS 8523, IRCICA, Université Lille 1, Villeneuve d'Ascq 59655, France \\ ${ }^{2}$ Optical Sciences Group, Research School of Physics and Engineering, \\ The Australian National University, Canberra ACT 0200, Australia
}

(Received 21 August 2013; revised manuscript received 16 December 2013; published 28 March 2014)

\begin{abstract}
The discovery of the Fermi-Pasta-Ulam (FPU) recurrence phenomenon in the $1950 \mathrm{~s}$ was a major step in science that later led to the discovery of solitons in nonlinear physics. More recently, it was shown that optical fibers can serve as a medium for observing the FPU phenomenon. In the present work, we have found experimentally and numerically that in the low-dispersion region of an optical fiber, the recurrence is strongly influenced by the third-order-dispersion (TOD) term. Namely, the presence of TOD leads to several disappearances and recoveries of the FPU recurrence when the central frequency of the pump wave is varied. The effect is highly nontrivial and can be explained in terms of reversible and irreversible losses caused by Cherenkov radiations interacting with a multiplicity of modes sharing the optical energy in the process of its partition.
\end{abstract}

DOI: 10.1103/PhysRevX.4.011054

\section{INTRODUCTION}

The phenomenon of Fermi-Pasta-Ulam (FPU) recurrence has attracted massive attention from researchers since the initially classified technical report of Fermi and his colleagues was published in 1955 (see Ref. [1]). FPU recurrence is a ubiquitous phenomenon observable in many fields of physics ranging from plasma physics [2] to hydrodynamics [3] and optics [4-6]. Specifically, Fermi et al. were investigating the dynamics of a vibrating chain of nonlinearly coupled identical oscillators [1]. An idea was to model thermalization processes taking place in a solid state. Because of the expected equipartition, the energy of the excited vibrational mode was reckoned to spread out equally between all other normal modes of the chain. Counterintuitively, numerical simulations showed a different dynamics: After the initial diffusion to other normal modes, energy returned to the same mode that was excited initially. This completely unexpected outcome of the numerical experiment is sometimes called the FPU paradox [7]. Later studies have shown that recurrence is not an exception but happens in a variety of physical systems of interest [2-4]. Remarkably, further attempts to solve the paradox [8] resulted in a subsequent great leap in sciencethe birth of the concept of solitons.

\footnotetext{
*Corresponding author. arnaud.mussot@univ-lille1.fr
}

Published by the American Physical Society under the terms of the Creative Commons Attribution 3.0 License. Further distribution of this work must maintain attribution to the author(s) and the published article's title, journal citation, and DOI.
Subject Areas: Nonlinear Dynamics, Optics

Among many fields of physics where the FPU recurrence may occur, fiber optics offer one of the best platforms to study the phenomenon, as all experiments are done with standard compact tabletop equipment, rather than complex experimental apparatus needed in plasma physics or hydrodynamics, for instance. The first experimental demonstration of the FPU recurrence in optical fibers was reported by Van Simaeys et al. [4-6] in the high-dispersion regime of a standard telecommunication fiber where the third-orderdispersion (TOD) term is negligible. In this configuration, the role played by higher-order linear terms is negligible, so the dynamics is well reproduced by the pure nonlinear Schrödinger equation (NLSE). As a consequence, analytic solutions known as Akhmediev breathers (ABs) [9] accurately describe the recurrence process. The latter can be considered as the extension of the modulation-instability process [10] beyond the linear approximation that captures the full nonlinear dynamics of the system. These exact solutions represent a fundamental tool for understanding optical phenomena that start with a strong monochromatic wave [4,5,11-15]. Among them, there were the first experimental observations of the Peregrine [16] and Kusnetzov-Ma [17] breathers, well after their first theoretical predictions [18-20].

The pure NLSE is valid for optical fields with relatively narrow spectra. If the dispersion cannot be considered to be a constant over the spectrum, the TOD term must be taken into account [21-28]. This higher-order term strongly modifies the dynamics of nonlinear systems by making them convectively unstable [21]. In optical fibers, it leads to the nonlinear symmetry breaking of parametric processes [22-24,28], to the generation of Cherenkov radiations 
(CRs) by solitons [29], which are one of the key elements of supercontinuum generation [30,31], and, in extreme cases, to turbulent dynamics $[32,33]$.

The FPU recurrence is also strongly affected by the TOD [26]. In Ref. [26], it was found that the FPU recurrence may disappear and appear again under its influence. The main reason for this behavior is the Cherenkov radiation that dissipates the energy of the pump into small-amplitude radiation waves. The present experiment has been set up in order to confirm this prediction. To our surprise, we have found a quite different behavior. Namely, we have discovered experimentally that the FPU recurrence experiences multiple appearances and disappearances-not only one-in contrast to the theoretical expectations of Ref. [26]. We have found that they are related to reversible and irreversible losses caused by the TOD.

Up to now, the multiple appearances and disappearances of the FPU recurrence caused by reversible and irreversible losses, respectively, have never been observed in physics. Among similar phenomena, we can mention the photonecho phenomenon, which is a physical effect where the radiation energy captured by a system of inhomogeneously broadened two-level atoms can be recovered and reemitted as a coherent optical pulse [34]. The effect that we have discovered here is another example of reversible losses in optics, although it is quite different from the optical echo. The uniqueness of this phenomenon lies in the multiple cancellations and recoveries of the FPU recurrence when the pump frequency, and consequently the groupvelocity dispersion, are varied. This new feature reveals significantly more complex dynamics than previously thought. Our experiment goes well beyond asymmetries observed in spectra via Cherenkov-radiation-assisted parametric resonances either in the context of frequency combs, as recently reported in Ref. [23], or in the framework of noise-seeded modulation instability (MI) fiber systems reported in Ref. [22], where random initial conditions prevent the amplification of a single resonant mode (a monochromatic wave in optics) that is crucial in the FPU concept [1]. We show here that the system is capable of recovering its initial state by reversing the energy-flow transfers, which was not the case either in Ref. [23] or in Ref. [22].

\section{EXPERIMENTAL SETUP AND METHODS}

Our experimental setup is depicted in Fig. 1(a). We use two monochromatic tunable diodes, producing the pump and signal waves shown in the upper left corner of Fig. 1. The two optical fields are coupled together through a polarization-maintaining 50/50 coupler. Each one is phase modulated with a pseudorandom bit sequence at $3 \mathrm{Gbits} / \mathrm{s}$ in order to avoid the stimulated Brillouin-scattering effect. The two waves are then amplified in an erbium-doped fiber amplifier (EDFA) before being launched into a dispersionshifted fiber (DSF). In order to reach perfect alignment between the polarization states of the pump and the signal waves before being launched into the DSF, the setup is equipped with polarization-maintaining components in front of the second coupler C2. The DSF is the same as in Ref. [22]. The combined power of the pump and the signal launched into the fiber is $27.7 \mathrm{dBm}$, and the difference between the pump and the signal is $11 \mathrm{~dB}$. In
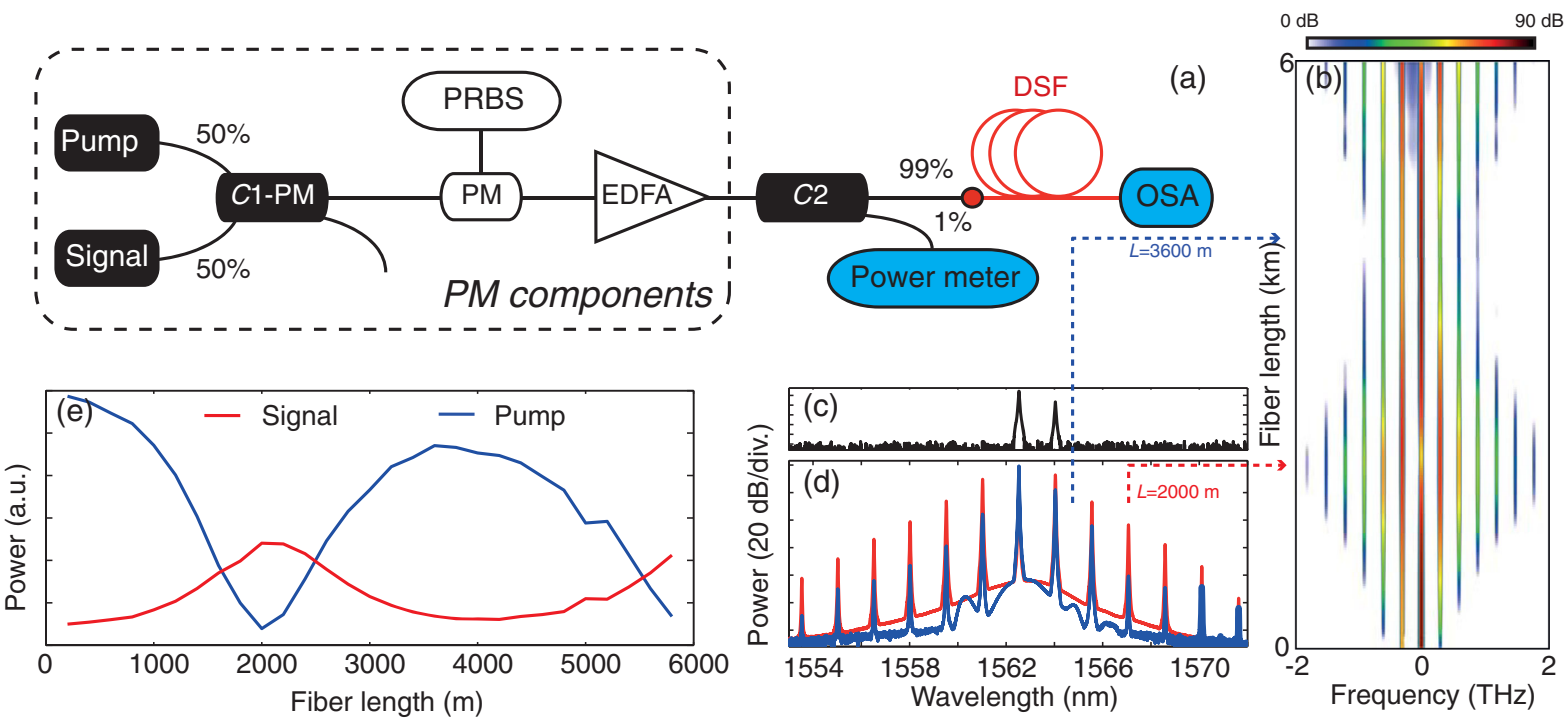

FIG. 1 (a) Experimental setup. Notations are as follows: $C_{1,2}$ are optical couplers; PRBS, pseudorandom bit sequence; PM, phase modulator; and OSA, optical spectrum analyzer. Experimental data: (b) Evolution of the spectrum versus fiber length. Spectra at specific lengths at (c) $L=0 \mathrm{~m}$ and (d) $L=2000 \mathrm{~m}$ (red line) and $L=3600 \mathrm{~m}$ (blue line). (e) Evolution of the pump and signal powers versus fiber length. All these experimental results have been obtained with a second-order-dispersion value $\beta_{2}=-1.116 \times 10^{-27} \mathrm{~s}^{2} / \mathrm{m}$ (11.7 $\mathrm{nm}$ above the fiber ZDW). 
other words, $P_{P}=530 \mathrm{~mW}$ and $P_{S}=44.7 \mathrm{~mW}$. In order to achieve the strongest MI gain, the signal wavelength is chosen at the maximum of the MI gain band. This corresponds to a value of $a=0.25$, if we refer to the dimensionless parameter $a$ used in the standard AB theory [16]. This wavelength is measured from the experimental MI spectra recorded without the signal launched into the fiber, i.e., assuming noisy initial conditions. We pay special attention to keeping the powers of the two waves constant during the wavelength sweep (maximum fluctuations of $0.3 \mathrm{~dB}$ ).

Before investigating the role played by TOD in the FPU process, we have made sure that our experimental setup allows us to observe the "standard" FPU recurrence in an optical fiber, as it was reported in Refs. [4,5]. In order to do so, first, we look for a configuration such that the role played by TOD is negligible. We fix the pump wavelength far away from the zero-dispersion wavelength (ZDW) of the fiber in order to have a relatively large dispersion over the whole spectrum, which can be assumed to be constant. Figure 1(b) represents the evolution of the spectrum versus the fiber length. An immediate observation is that the spectrum is almost symmetric at each side of the pump wavelength, as expected from the theory based on the NLSE equation [9]. This observation confirms that we have achieved the configuration where the TOD does not significantly affect the process. The second essential observation is that the MI process naturally turns into the FPU recurrence as fiber length increases. The energy that was initially confined to the pump and the signal waves [see the input spectrum in Fig. 1(c)] is progressively transferred to the signal's harmonics on each side of the pump [Figs. 1(b) and 1(d)]. To get a better insight, Fig. 1(d) represents the evolution of the signal and the pump powers versus fiber length. A maximum energy-transfer rate of $92 \%$ with $8 \%$ left in the pump is reached at $L=2000 \mathrm{~m}$. At this position, the signal power is maximal and the spectrum is the broadest, with more than six harmonics generated on each side of the spectrum [see the red trace in Fig. 1(d)]. The energy transfer is stopped and further reversed, starting from this fiber length. It then flows from the harmonics back to the pump. It reaches about $79 \%$ of the initial pump power at $L=3600 \mathrm{~m}$ [see Fig. 1(e) and the blue trace in Fig. 1(d)].

This high value illustrates that the FPU process is very efficient. Indeed, by only accounting for the linear losses, we would find the pump power after propagating $3600 \mathrm{~m}$ inside the fiber to be equal to $84 \%$ of the initial value. After one period of this process, the energy of the wave flows again from the pump to the sidebands, starting a second cycle of recurrence. We are able to observe more than oneand-a-half periods of recurrence, just as in the original work of Van Simaeys et al. This result is a clear and clean signature of the FPU recurrence phenomenon. Now, after the confirmation of the existence of FPU recurrence, we can move to the observation of the impact of the TOD on it. In order to do so, the pump wavelength has been shifted toward the ZDW of the fiber. As a consequence, MI spectra, with the width being inversely proportional to the secondorder dispersion, will be broadened, and it will no longer be possible to consider the dispersion over the whole spectral span to be constant. It will thus be necessary to account for the TOD term, as in other studies in nonlinear fiber optics related to MI [22,24,25] in the low-dispersion region of optical fibers.

\section{RESULTS}

The pump wavelength is tuned between $1561.6 \mathrm{~nm}$ and the ZDW of the fiber $(1550.2 \mathrm{~nm})$ with 61 steps and the spectra recorded every $200 \mathrm{~m}$ after successive cutbacks. This tuning range causes $\beta_{2}$ to vary from $\beta_{2}=-12 \times 10^{-28} \mathrm{~s}^{2} / \mathrm{m}$ to 0 . In this relatively short-wavelength span, the slope of the dispersion can be assumed to be constant, and tuning the pump wavelength, i.e., the dispersion value, is equivalent to varying the ratio $\beta_{3} / \beta_{2}$. Figures 2 (a) and 2(b) represent the evolution of the pump power and the signal powers, respectively, along the fiber for these $\left|\beta_{2}\right|$ values. These data are presented in the form of a 3D plot with "hot" colors corresponding to the highest powers. It is worth noting that the usual FPU recurrence process [where TOD is negligible; see Fig. 1(e)] corresponds to the highest value of $\left|\beta_{2}\right|$. A comparable behavior with this process can still be observed by decreasing $\left|\beta_{2}\right|$ up to about $6 \times 10^{-28} \mathrm{~s}^{2} / \mathrm{m}$, where more than one-and-a-half periods of recurrence still occur.

Thus, in this range, the contribution of $\beta_{3}$ is much smaller than that of $\beta_{2}$, and it does not significantly affect the FPU recurrence process. However, as $\left|\beta_{2}\right|$ further decreases (as the pump wavelength shifts toward the ZDW in our experiments), the TOD becomes significant. A much more complex dynamics is then observed [see Figs. 2(a) and 2(b)].

In order to explain this behavior, we show in Figs. 2(e) three striking examples. In Fig. 2(e) $[\alpha]$, the pump- and signal-power evolutions are very similar to the reference curves given in Fig. 1(e). The FPU recurrence is clearly visible with more than one-and-a-half periods. By slightly decreasing the absolute value of the second-order dispersion to $\left|\beta_{2}\right|=2.78 \times 10^{-28} \mathrm{~s}^{2} / \mathrm{m}$, we can see in Fig. $2(\mathrm{e})[\beta]$ that once the pump power is transferred to the normal modes, the reversed process does not occur, and the pump power remains fixed around this lower value. We can then conclude that the recurrence process is canceled for this specific value of $\left|\beta_{2}\right|$. However, a further slight decrease of the $\left|\beta_{2}\right|$ value to $2.4 \times 10^{-28} \mathrm{~s}^{2} / \mathrm{m}$ restores the FPU recurrence again, as shown in Fig. 2(e) $[\chi]$. As can be seen from Figs. 2(a) and 2(b), this oscillating behavior is repeated several times when reducing the value of the $\left|\beta_{2}\right| / \beta_{3}$ ratio. More details of the evolution of the pump and signal powers for each value that switches the FPU recurrence on and off can be found in the Appendix. 

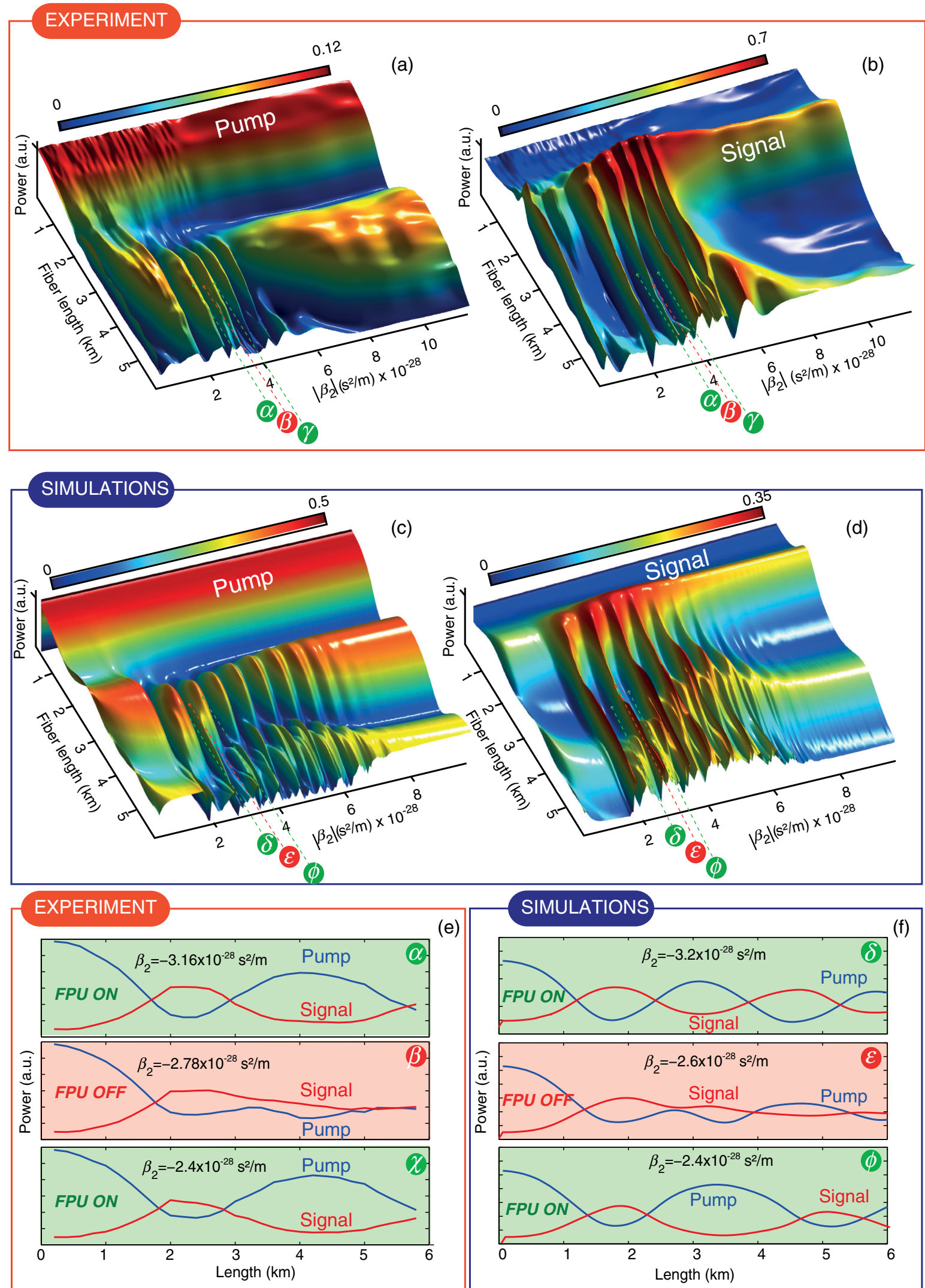

(e)

\section{SIMULATIONS}

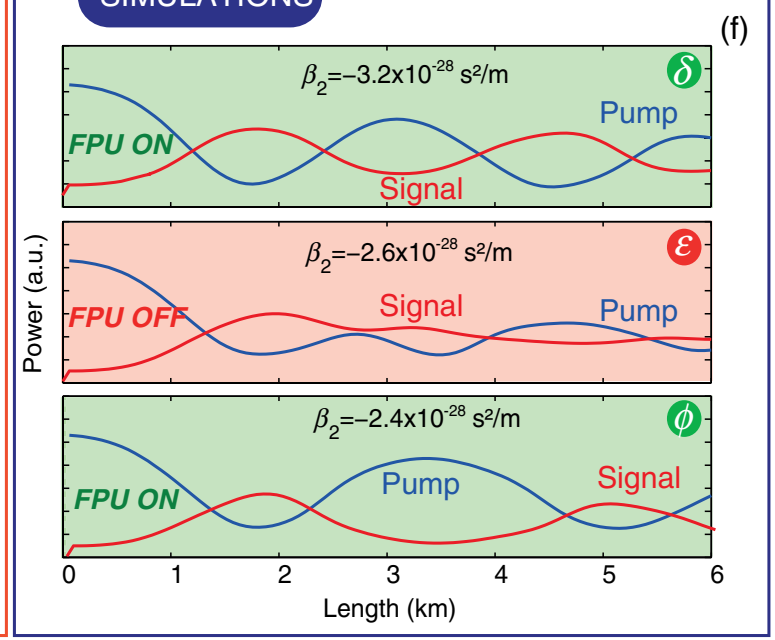

FIG. 2 Plot of the pump and the signal power versus $\left|\beta_{2}\right|$ and the fiber length, (a) and (b) in experiments and (c) and (d) in simulations. (e) and (f) show their evolution at specific $\left|\beta_{2}\right|$ values for which the FPU recurrence is observed or canceled, in experiments and simulations, respectively. 
These experimental results are well supported, with realistic numerical simulations performed by integrating the generalized nonlinear Schrödinger equation

$$
\begin{aligned}
\frac{\partial E(z, \tau)}{\partial z}= & -i \frac{\beta_{2}}{2} \frac{\partial^{2} E(z, \tau)}{\partial \tau^{2}}+\frac{\beta_{3}}{6} \frac{\partial^{3} E(z, \tau)}{\partial \tau^{3}}+i \frac{\beta_{4}}{24} \frac{\partial^{4} E(z, \tau)}{\partial \tau^{4}} \\
& +i \gamma|E|^{2} E(z, \tau)+i \gamma \int h_{R}(t)\left|E\left(z, \tau-\tau^{\prime}\right)\right|^{2} d \tau^{\prime} E(z, \tau),
\end{aligned}
$$

where $\beta_{2,3,4}$ are the second-, third-, and fourth-orderdispersion terms, respectively, $\gamma$ is the fiber nonlinear coefficient, and $h_{R}(t)$ is the Raman response, taken from the experimental measurements. It has been numerically integrated using an adaptive split-step Fourier method [35], with a precision of $10^{-7}$. We use the following parameters, which are close to the experimental ones: $\beta_{2}\left(\lambda_{P}\right)=-100 \times 10^{-29}$ to $-1 \times 10^{-29} \mathrm{~s}^{2} / \mathrm{m}$ (200 steps), $\beta_{3}\left(\lambda_{P}\right)=1.2 \times 10^{-40} \mathrm{~s}^{3} / \mathrm{m}, \quad \beta_{4}\left(\lambda_{P}\right)=-2.5 \times 10^{-55} \mathrm{~s}^{4} / \mathrm{m}$, $\gamma=2.4 / \mathrm{W} / \mathrm{km}$, fiber length $L=6000 \mathrm{~m}$, linear attenuation $\alpha=0.2 \mathrm{~dB} / \mathrm{km}, P_{P}=530 \mathrm{~mW}$, and $P_{S}=44.7 \mathrm{~mW}$. Numerical results are summarized in Figs. 2(c), 2(d), and 2(f). While no adjustable parameter has been used, very good agreement is achieved, apart from the fact that the beginning of the second period of recurrence occurrs at $L=3000 \mathrm{~m}$ in simulations against $L=4000 \mathrm{~m}$ in experiments. This slight discrepancy may be explained by inaccuracies in the pump-power measurements and/or due to longitudinal fluctuations of the dispersion and/or polarization-mode dispersion in the actual fiber. In a previous theoretical work performed by one of us in Ref. [26], the FPU recurrence process was expected to disappear under the action of the slope of the dispersion and to appear again in very low-dispersion regions. In our present work, we find that the dynamics is in fact much more complex, since there is a range of $\left|\beta_{2}\right|$ values where it appears and disappears many times. This remarkable feature has never been observed in any other physical system, and it shows that, against intuition, such complex parametric processes of FPU recurrence also depend on the TOD.

\section{DISCUSSION}

In order to get a clearer insight into the multiple disappearances and restorations of the FPU recurrence, we plot them in Fig. 3(a) for a fixed fiber length. The data are taken from numerical results shown in Figs. 2(c) and 2(d) at $L=4000 \mathrm{~m}$, a length for which the FPU recurrence has the strongest contrast. This figure clearly illustrates the multiple disappearances and restorations of the FPU recurrence when the $\left|\beta_{2}\right|$ value is varied. For instance, FPU is on for each maximum of the pump and it exhibits one-and-a-half periods of recurrence in all these cases. (See the figures in the Appendix with a green background.) In order to understand the origin of this behavior, let us remind ourselves that the frequency of the signal has been fixed at the maximum of the MI gain spectrum. Its frequency shift from the pump can then be expressed as follows: $\Delta \omega_{\mathrm{MI}}=\omega_{p}-\omega_{s}=\sqrt{-2 \gamma p / \beta_{2}\left(\omega_{p}\right)}$. In Ref. [26], it has been demonstrated that under the action of TOD, Akhmediev breathers shed energy to smallamplitude Cherenkov radiations. This process happens under the following phase-matching condition: $\beta_{3} \Delta \omega_{\mathrm{CR}}^{3}+$ $3 \beta_{2} \Delta \omega_{\mathrm{CR}}^{2}-6 \gamma p=0$, where $\Delta \omega_{\mathrm{CR}}$ is the frequency shift of Cherenkov radiations from the pump. A combination of these two equations provides the values that are plotted in Fig. 3(a) using vertical dash-dotted green lines. These lines correspond to the cases when Cherenkov radiations are generated exactly on MI harmonics, i.e., when $\Delta \omega_{\mathrm{CR}}=$ $n \times \Delta \omega_{\mathrm{MI}}$, with $n$ an integer. Each of these lines is close to

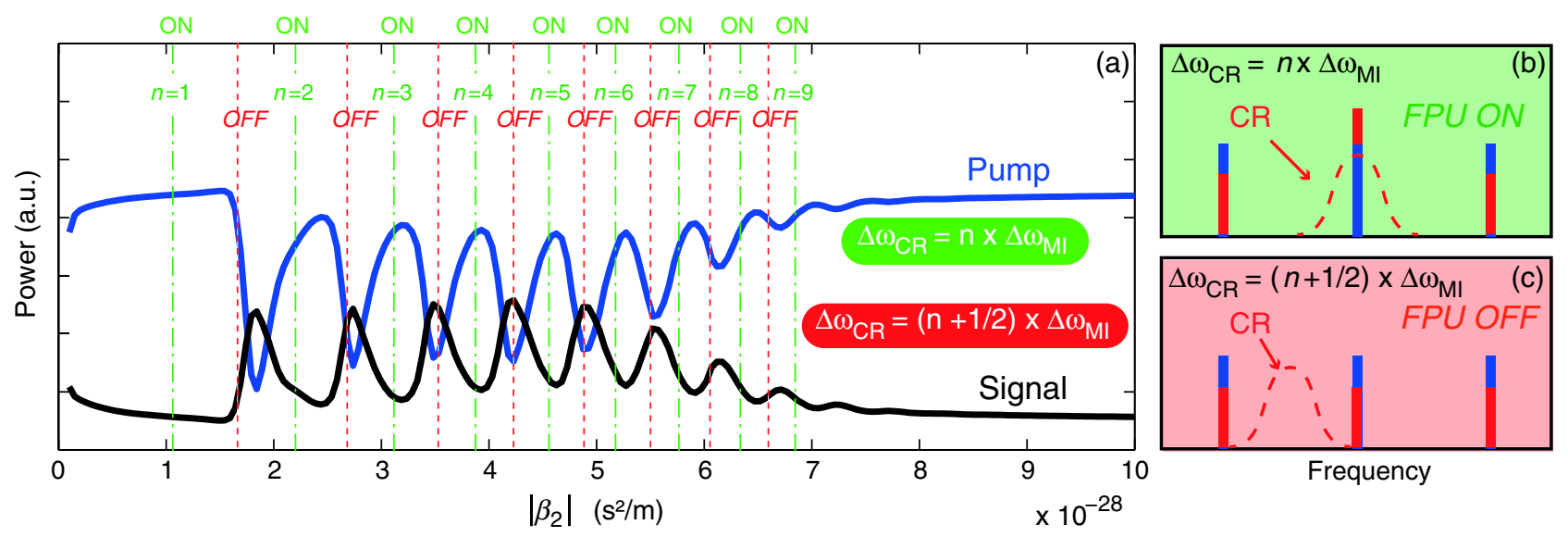

FIG. 3 (a) Evolution of the pump (solid blue curve) and first harmonics (solid black curve) powers versus second-order-dispersion values. Dash-dotted vertical green lines represent the $\left|\beta_{2}\right|$ values for which the Cherenkov radiations are exactly located on MI harmonics, while the dotted red lines represent the $\left|\beta_{2}\right|$ values when CRs are in the middle between the two harmonics. "ON" at the tops of the lines refers to the $\left|\beta_{2}\right|$ values for which the FPU recurrence is maximal. (b) The consequence of perfect phase matching between the MI harmonics and CRs. (c) The case of the phase mismatch. In (b) and (c), the dashed curves show the spectra of the CRs. Spectral components shown as blue bars are those before the CR generation, while red bars are those after the CR generation. 
one of the maxima of the pump power [blue curve in Fig. 3(a)]. In this case, the FPU is on and it is not affected by the TOD.

On the other hand, the vertical dashed red lines represent the cases in which the radiation modes fall exactly in the middle between the two MI harmonics $\left[\Delta \omega_{\mathrm{CR}}=\right.$ $\left.(n+0.5) \times \Delta \omega_{\mathrm{MI}}\right]$. Each of these lines is close to one of the minima of the pump power [blue curve in Fig. 3(a)], meaning that the FPU recurrence disappears. This simple observation can be explained as follows. When Cherenkov radiations are generated exactly on MI harmonics, it leads to a modification of the energy repartition in the spectrum, but most of the energy transferred to Cherenkov radiations remains within the MI frequency comb. Figure 3(b)
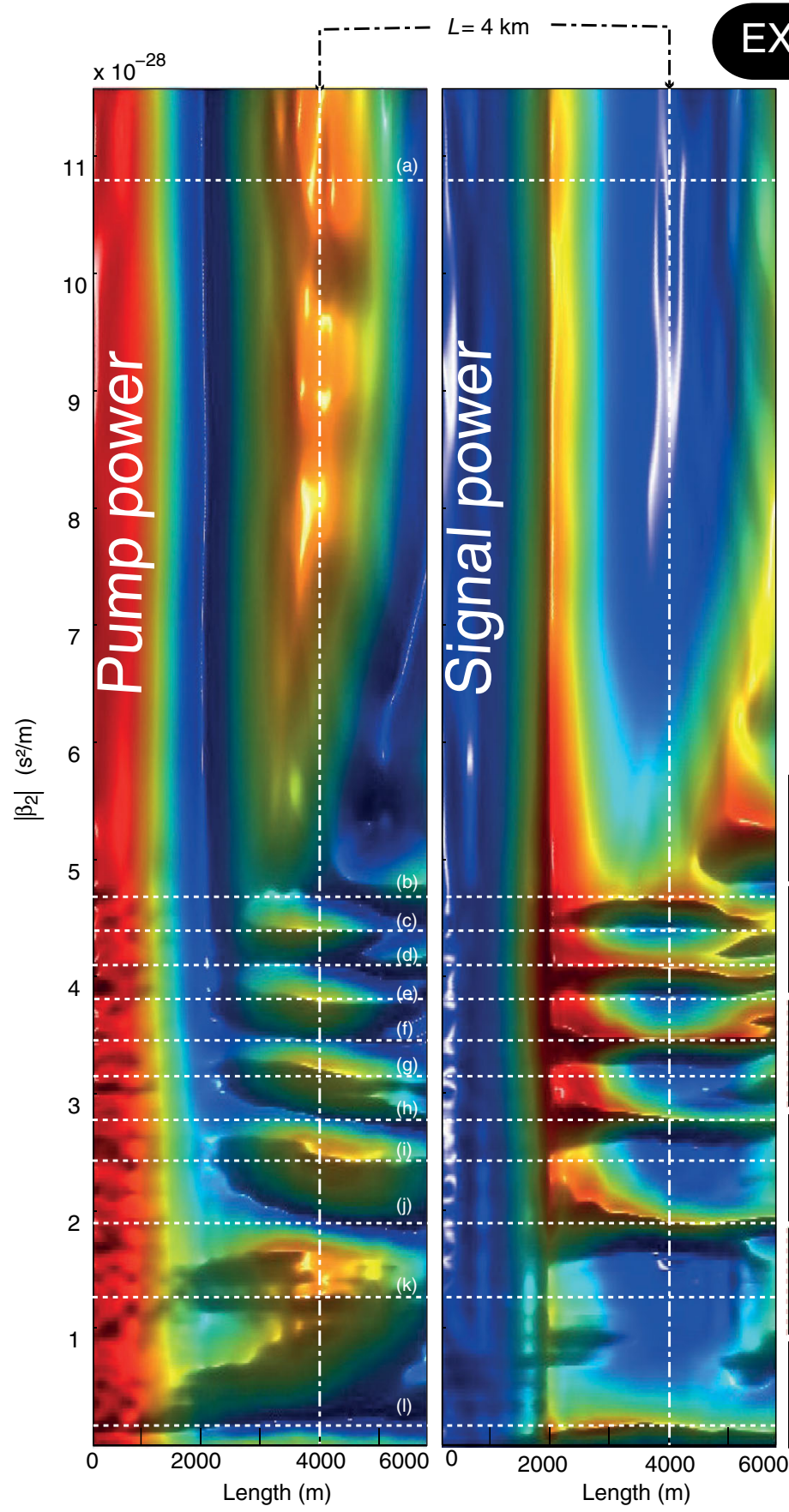

\section{EXPERIMENT}
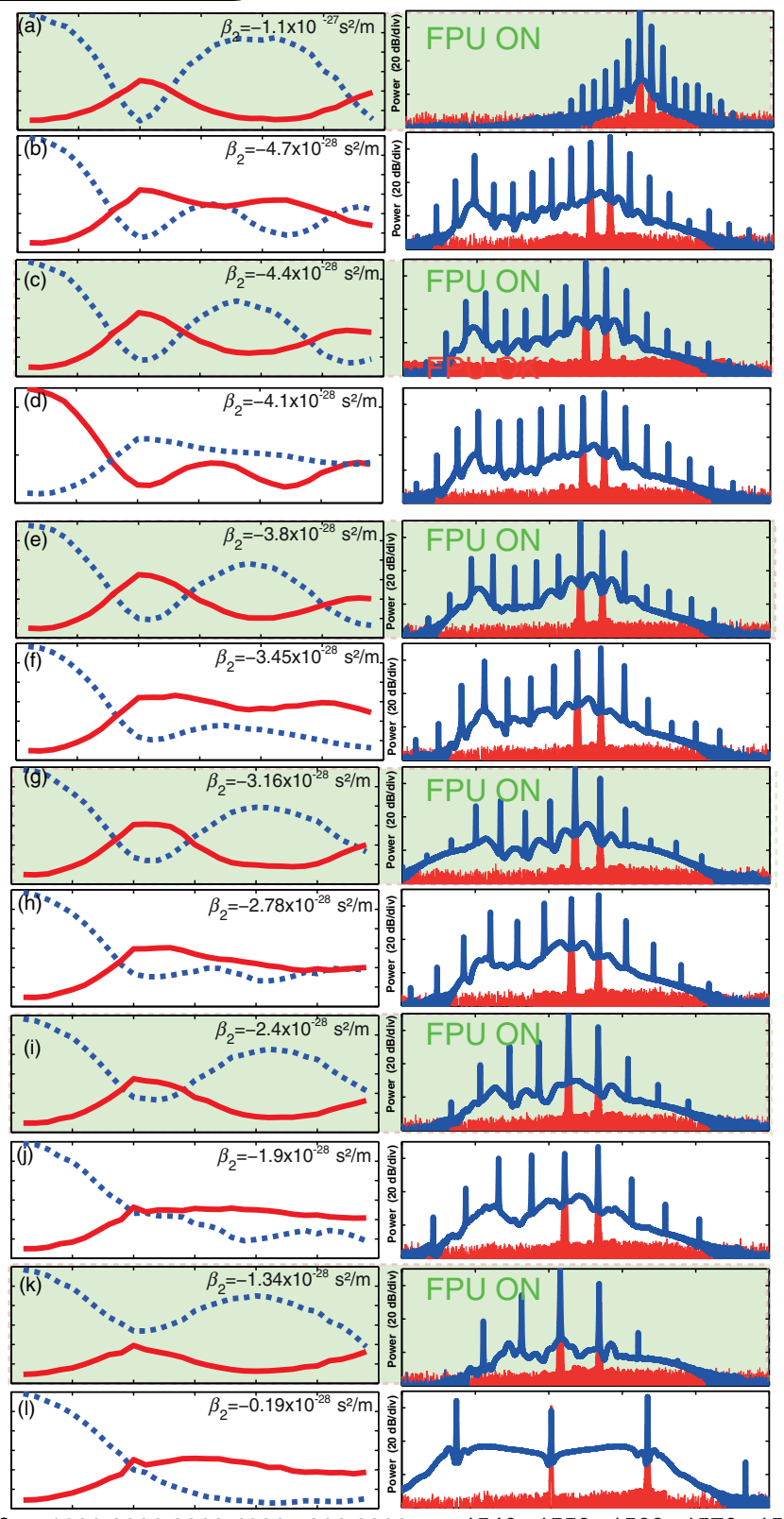

100020003000400050006000

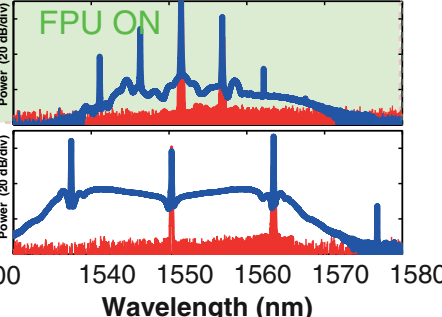

FIG. 4 Experimental data. Left two columns: False color plots of the pump and signal powers versus fiber length and dispersion. The third column: Evolution of the signal power (solid red lines) and of the pump power (dotted blue lines) versus fiber length for specific $\left|\beta_{2}\right|$ values. Right column: Corresponding spectra at $L=4000 \mathrm{~m}$ (blue lines) and $L=0$ (red lines). The pink panels in the two right columns show the cases where FPU recurrence is on, while the white panels show the cases where FPU recurrence is off. 
illustrates schematically this process. Then, multiple fourwave-mixing processes involved in the FPU recurrence may allow the energy of these normal modes to flow back to the pump. Thus, the losses here caused by Cherenkov radiations are reversible. On the contrary, when Cherenkov radiations are generated in between two successive MI harmonics, most of their energy is lost and is not recoverable for multiple four-wave-mixing processes [see the scheme in Fig. 3(c)]. The losses here are thus irreversible, and it leads to the suppression of the FPU recurrence due to the generation of Cherenkov radiations.

This procedure leads to multiple disappearances and restorations of the FPU recurrence, up to an upper limit (from about $\left|\beta_{2}\right|>8 \times 10^{-27} \mathrm{~s}^{2} / \mathrm{m}$ in our case). From this

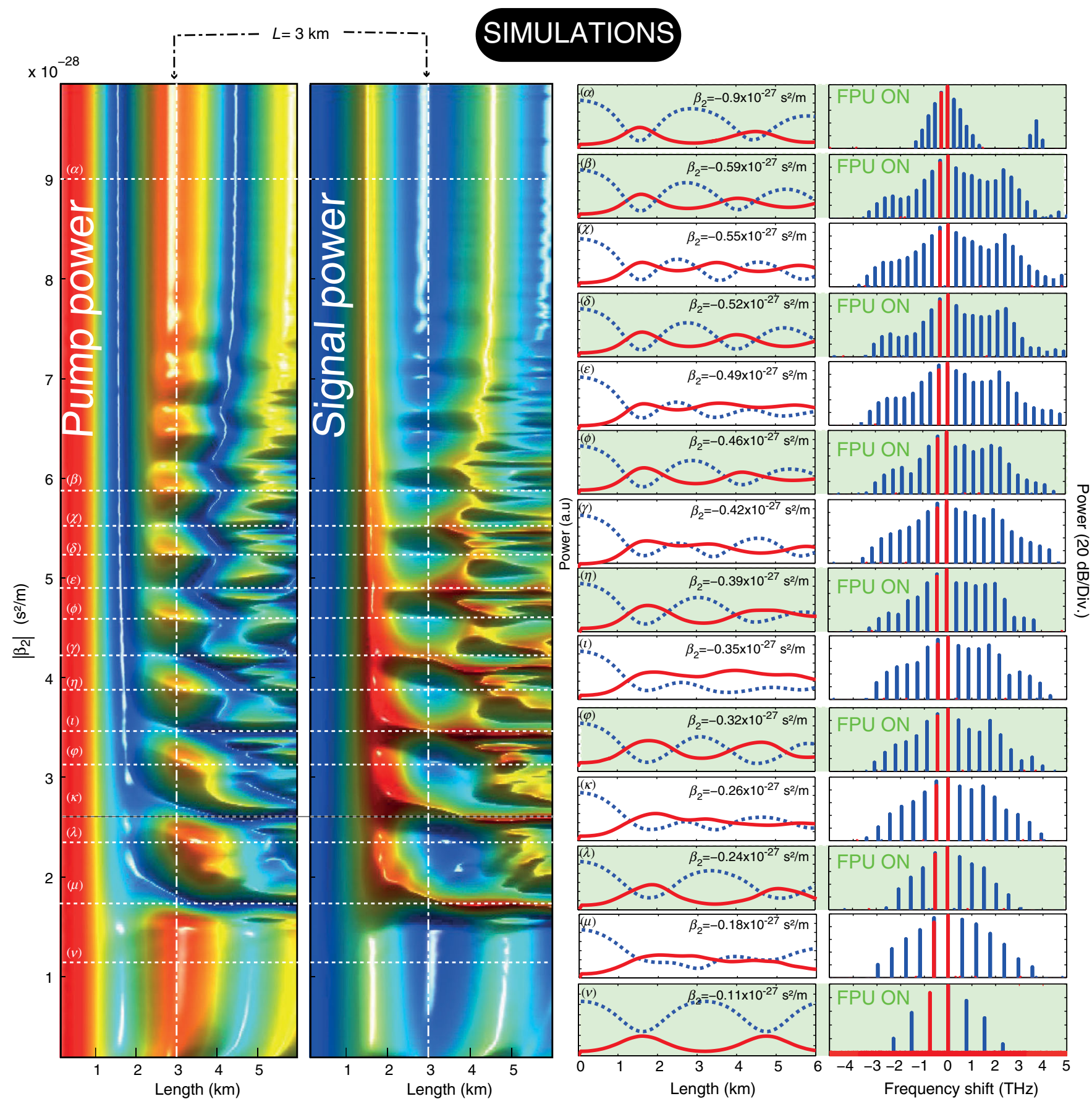

FIG. 5 Numerical data. Left two columns: False color plots of the pump and signal powers versus fiber length and dispersion. The third column: Evolution of the signal power (solid red lines) and of the pump power (dotted blue lines) versus fiber length for specific values of dispersion. Right column: Corresponding spectra at $L=3000 \mathrm{~m}$ (blue bars) and $L=0$ (red bars). Light pink panels in the two right columns show the cases where FPU recurrence is on, while the white panels show the cases where FPU recurrence is off. 
value, whatever the $\Delta \omega_{\mathrm{CR}} / \Delta \omega_{\mathrm{MI}}$ ratio, we do not observe any deviations from the standard FPU recurrence dynamics because the energy shed into Cherenkov radiations is very small [29]. The impact of Cherenkov radiations (and thus of the TOD) on the whole system is then negligible.

This behavior is not only valid for signals located at the maximum of the MI gain spectrum but similar behavior is expected for any other frequency shifts, i.e., for other values of the parameter a used in the $A B$ theory [16], as long as the energy shed by CRs is strong enough and the spectral width of CRs is lower than the frequency shift between two MI harmonics. In both cases, it corresponds to $a \ll 0.5$.

\section{CONCLUSION}

In conclusion, we have shown theoretically, numerically, and experimentally, for the first time to our knowledge, that FPU recurrence can be observed in an optical fiber with the remarkable feature of multiple disappearances and restorations due to third-order dispersion. These noteworthy features have been observed when the optical frequency changes in a small interval close to the zero-dispersion wavelength of the fiber. We have found that this behavior is related to the Cherenkov-radiation process and, more specifically, to the resonance between these smallamplitude radiation waves generated due to third-order dispersion and the MI harmonics. In the presence of perfect phase matching, the radiation into the MI harmonics is reversible, thus providing favorable conditions for the FPU recurrence to occur. On the other hand, the irreversible dissipation of energy into the radiation modes (when CRs fall in between two successive MI harmonics) causes the FPU recurrence to disappear. As far as we know, the effect that we have discovered here has never yet been observed in physics. The only other comparable case is the photon-echo phenomenon that allows us to restore optical energy coherently when it is lost within a system of two-level atoms. In the latter case, the system can be "reversed" in time by an external pulse, while the restoration of optical energy in the FPU phenomenon is caused by the coherent interaction of Cherenkov radiation with a multiplicity of MI sidebands. We anticipate that the effect could be observed in other convective systems that are modeled by the NLSE with higher-order-dispersion terms. In particular, it should be observable in the case of water waves or plasma.

\section{ACKNOWLEDGMENTS}

We acknowledge support from the French Agence Nationale de la Recherche (ANR-jeune chercheur FOPAFE and TOPWAVE), the Ministry of Higher Education and Research, Nord-Pas de Calais Regional Council, and FEDER through the Contrat de Projets Etat Région (CPER) 2007-2013. N. A. acknowledges the support of the Australian Research Council (Discovery
Projects No. DP110102068 and No. DP140100265). We thank Olivier Vanvincq for fruitful discussions and Dr. Ankiewicz for careful reading of the manuscript.

\section{APPENDIX}

Figure 4 provides additional information related to experimental results.

The two left panels in this figure represent the top view of Figs. 3(a) and 3(b), and the third column represents the evolution of the pump and signal powers (in dashed blue lines and solid red lines, respectively) for specific secondorder-dispersion values. The chosen values correspond to the extrema of the pump power in Fig. 4(a) at a distance $L=3000 \mathrm{~m}$, where the FPU contrast is maximal. When the pump power reaches a maximum value, the FPU recurrence is on. This fact is highlighted by coloring the corresponding panels in pink. The right panel in Fig. 4 shows the initial $(L=0)$ and the final $(L=4000 \mathrm{~m})$ spectra at the same values of $\left|\beta_{2}\right|$ as in the third panel.

Similar data taken from numerical simulations are presented in Fig. 5. The qualitative features of this plot are the same as in Fig. 4.

[1] E. Fermi, J. Pasta, and S. Ulam, Collected Papers of Enrico Fermi, edited by E. Segre (Literary Licensing, Italy, 1965), p. 978-988.

[2] T. Taniuti and H. Washimi, Self-Trapping and Instability of Hydromagnetic Waves along the Magnetic Field in a Cold Plasma, Phys. Rev. Lett. 21, 209 (1968).

[3] T. B. Benjamin and J. E. Feir, The Disintegration of Wave Trains on Deep Water Part 1. Theory, J. Fluid Mech. 27, 417 (1967).

[4] G. Van Simaeys, P. Emplit, and M. Haelterman, Experimental Demonstration of the Fermi-Pasta-Ulam Recurrence in a Modulationally Unstable Optical Wave, Phys. Rev. Lett. 87, 033902 (2001).

[5] G. Van Simaeys, P. Emplit, and M. Haelterman, Experimental Study of the Reversible Behavior of Modulational Instability in Optical Fibers, J. Opt. Soc. Am. B 19, 477 (2002).

[6] N. N. Akhmediev, Nonlinear Physics: Déjá Vu in Optics, Nature (London) 413, 267 (2001).

[7] The Fermi-Pasta-Ulam Problem: A Status Report, edited by G. Gallavotti (Springer, New York, 2007), 1st ed.

[8] N. J. Zabusky and M. D. Kruskal, Interaction of "Soliton$s$ "in a Collisionless Plasma and the Recurrence of Initial States, Phys. Rev. Lett. 15, 240 (1965).

[9] N. N. Akhmediev and V. I. Korneev, Modulation Instability and Periodic Solutions of the Nonlinear Schrödinger Equation, Teor. Mat. Fiz. 69, 189 (1986).

[10] V. E. Zakharov and L. A. Ostrovsky, Modulation Instability: The Beginning, Physica (Amsterdam) 238D, 540 (2009).

[11] N. Akhmediev, J. M. Soto-Crespo, and A. Ankiewicz, Extreme Waves That Appear from Nowhere: On the Nature of Rogue Waves, Phys. Lett. A 373, 2137 (2009). 
[12] N. Akhmediev and E. Pelinovsky, Editorial-Introductory Remarks on Discussion \& Debate: Rogue Waves-Towards a Unifying Concept?, Eur. Phys. J. Spec. Top. 185, 1 (2010).

[13] M. Erkintalo, G. Genty, B. Wetzel, and J. M. Dudley, Akhmediev Breather Evolution in Optical Fiber for Realistic Initial Conditions, Phys. Lett. A 375, 2029 (2011).

[14] M. Erkintalo, K. Hammani, B. Kibler, C. Finot, N. Akhmediev, J. M. Dudley, and G. Genty, Higher-Order Modulation Instability in Nonlinear Fiber Optics, Phys. Rev. Lett. 107, 253901 (2011).

[15] N. Akhmediev, A. Ankiewicz, and M. Taki, Waves That Appear from Nowhere and Disappear without a Trace, Phys. Lett. A 373, 675 (2009).

[16] B. Kibler, J. Fatome, C. Finot, G. Millot, F. Dias, G. Genty, N. Akhmediev, and J. M. Dudley, The Peregrine Soliton in Nonlinear Fibre Optics, Nat. Phys. 6, 790 (2010).

[17] B. Kibler, J. Fatome, C. Finot, G. Millot, G. Genty, B. Wetzel, N. Akhmediev, F. Dias, and J. M. Dudley, Observation of Kuznetsov-Ma Soliton Dynamics in Optical Fibre, Sci. Rep. 2, 463 (2012).

[18] Y.-C. Ma, The Perturbed Plane-Wave Solutions of the Cubic Schroedinger Equation, Stud. Appl. Math. 60, 43 (1979).

[19] E. A. Kuznetsov, Solitons in a Parametrically Unstable Plasma, Sov. Phys. Dokl. 22, 507 (1977).

[20] D. H. Peregrine, Water Waves, Nonlinear Schrödinger Equations and Their Solutions, J. Aust. Math. Soc. Series B, Appl. Math. 25, 16 (1983).

[21] A. Mussot, E. Louvergneaux, N. Akhmediev, F. Reynaud, L. Delage, and M. Taki, Optical Fiber Systems Are Convectively Unstable, Phys. Rev. Lett. 101, 113904 (2008).

[22] M. Droques, B. Barviau, A. Kudlinski, M. Taki, A. Boucon, T. Sylvestre, and A. Mussot, Symmetry-Breaking Dynamics of the Modulational Instability Spectrum, Opt. Lett. 36, 1359 (2011).

[23] M. Erkintalo, Y. Q. Xu, S. G. Murdoch, J. M. Dudley, and G. Genty, Cascaded Phase Matching and Nonlinear Symmetry Breaking in Fiber Frequency Combs, Phys. Rev. Lett. 109, 223904 (2012).
[24] Z. Lali-Dastjerdi, K. Rottwitt, M. Galili, and C. Peucheret, Asymmetric Gain-Saturated Spectrum in Fiber Optical Parametric Amplifiers, Opt. Express 20, 15530 (2012).

[25] A. Mussot, E. Lantz, H. Maillotte, T. Sylvestre, C. Finot, and S. Pitois, Spectral Broadening of a Partially Coherent CW Laser Beam in Single-Mode Optical Fibers, Opt. Express 12, 2838 (2004).

[26] J. M. Soto-Crespo, A. Ankiewicz, N. Devine, and N. Akhmediev, Modulation Instability, Cherenkov Radiation, and Fermi-Pasta-Ulam Recurrence, J. Opt. Soc. Am. B 29, 1930 (2012).

[27] C. Michel, P. Suret, S. Randoux, H. R. Jauslin, and A. Picozzi, Influence of Third-Order Dispersion on the Propagation of Incoherent Light in Optical Fibers, Opt. Lett. 35, 2367 (2010).

[28] F. Leo, A. Mussot, P. Kockaert, P. Emplit, M. Haelterman, and M. Taki, Nonlinear Symmetry Breaking Induced by Third-Order Dispersion in Optical Fiber Cavities, Phys. Rev. Lett. 110, 104103 (2013).

[29] N. Akhmediev and M. Karlsson, Cherenkov Radiation Emitted by Solitons in Optical Fibers, Phys. Rev. A 51, 2602 (1995).

[30] J. M. Dudley and J. R. Taylor, Supercontinuum Generation in Optical Fibers (Cambridge University Press, Cambridge, England, 2010), 1 ed.

[31] D. V. Skryabin and A. V. Gorbach, Colloquium: Looking at a Soliton through the Prism of Optical Supercontinuum, Rev. Mod. Phys. 82, 1287 (2010).

[32] G. Genty, C. M. de Sterke, O. Bang, F. Dias, N. Akhmediev, and J. M. Dudley, Collisions and Turbulence in Optical Rogue Wave Formation, Phys. Lett. A 374, 989 (2010).

[33] K. Hammani, B. Kibler, C. Finot, and A. Picozzi, Emergence of Rogue Waves from Optical Turbulence, Phys. Lett. A 374, 3585 (2010).

[34] L.C. Allen, Optical Resonance and Two-Level Atoms (Courier Dover Publications, New York, 1987).

[35] O. V. Sinkin, R. Holzlohner, J. Zweck, and C. R. Menyuk, Optimization of the Split-Step Fourier Method in Modeling Optical-Fiber Communications Systems, J. Lightwave Technol. 21, 61 (2003). 